\title{
Facilitation and interference with a separable redundant dimension in stimulus comparison
}

\author{
W. R. GARNER \\ Yale University, New Haven, Connecticut
}

\begin{abstract}
A comparison experiment was carried out with four letter stimuli such that some of the letter pairs provided hard and others easy discrimination when the pairs were different. A control condition confirmed the differences in difficulty with black letters. Two other conditions used two letters in each of two colors. When letter discrimination was hard, this redundant dimension produced facilitation of reaction time when the colors differed and were thus compatible with the "different" response required by the letters, and interference when the colors were the same, thus being incompatible with the "different" response. With easy letter pairs, only interference was found with incompatible color. Two additional experiments using classification procedures found color to have very little effect. Previous experiments had also shown different results concerning the separability or integrality of stimulus dimensions with the classification and comparison procedures. The interpretation for the difference suggested here is that response compatibility is an inherent aspect of the comparison procedure, since any dimension can have levels that are the same or different. The compatibility relation is available for only a few dimensional pairs with the classification procedure. When compatibility exists with either procedure, both interference and facilitation can occur, depending on whether the response interactions are compatible or incompatible.
\end{abstract}

The answers to many experimental questions do not appear to depend on the specific experimental paradigm or procedure used: the results are task-independent. In one problem area, however, that defining the difference between integral and separable dimensions based on reaction time (RT) measures, the outcomes appear to be taskdependent. Santee and Egeth (1980) have made the distinction most sharply, showing, for instance, that the two dimensions of shape and brightness are separable with a speeded classification procedure but appear to act integrally with a comparison (same-different) procedure. The purpose of the present report is to explore why this difference in experimental outcomes occurs.

\section{Integrality with Speeded Classification}

The typical experiment with speeded classification (see Garner, 1974, for more detail) uses four stimuli generated by the crossing of two dichotomous dimensions to produce an orthogonal set of stimuli. Thus, if form and color are the dimensions, there would be, to illustrate, a red square, a green square, a red circle, and a green circle. The experimental procedure involves the presentation of one stimulus at a time, and the subject is required to make a dichotomous response. Three different types of task are used: (1) Simple discrimination, in which just two stimuli varying on a single dimension are used, the

This research was supported by National Institutes of Health Grant MH-14229 to Yale University. Carolyn Paul carried out all of the experiments, helped design them, and did most of the data analysis. Her invaluable assistance is gratefully acknowledged. Requests for reprints should be sent to W. R. Garner, Department of Psychology, Box 11-A Yale Station, New Haven, CT 06520-7447. other dimension being constant. There are four such tasks, two for each dimension, and they serve as the control tasks. (2) Correlated discrimination, in which a pair of stimuli is again used, but the pair differs in both dimensions. Thus the dimensions are correlated. There are two such tasks, one for each possible pairing. (3) Full classification, in which the four orthogonal stimuli are used and the subject is required to classify the stimuli on the basis of one specified relevant dimension, the other dimension being irrelevant to the task. There are two such tasks, one for each dimension, as relevant.

The experimental outcome defining integral dimensions is that correlated discrimination is faster than simple or control discrimination, and that full classification is slower than simple discrimination. The result with correlation discrimination is generally interpreted to mean that discrimination is improved by the subject's use of both dimensions. The result with full classification is generally interpreted as a failure of selective attention to dimensions. These results together mean that the two dimensions act as one; that is, they are integral.

The experimental outcome defining separable dimensions is that correlated dimensions provide no improvement in discrimination, but full classification is as fast as simple discrimination. The failure of a correlation gain is interpreted to mean that the dimensions cannot be used jointly. The result with full classification is generally interpreted as meaning that selective attention to dimensions is possible with separable dimensions. Most, but not all, of the research comparing the differences in the classification and comparison procedures has centered on the selective attention aspect of the problem. 
Integrality with Stimulus Comparison

Differentiating integral from separable dimensions is quite straightforward with the speeded classification tasks, and these were the original speeded tasks used in defining the difference (Garner, 1970, 1974, 1976; Garner \& Felfoldy, 1970). With the comparison task, both the task and its interpretation concerning the distinction are much more complicated. The most elementary use and interpretation of the task is this: The four stimuli are each paired with themselves and the other three stimuli, forming 16 pairs of stimuli altogether. With one dimension specified as relevant and the other as irrelevant, these 16 pairs fall naturally into four groups of 4 pairs each.

For purposes of illustration, form will be considered the relevant dimension, with levels square (S) and circle (C), and color the irrelevant dimension, with levels red (R) and green (G). The four groups of pairs are: (A) SR-SR, SG-SG, CR-CR, CG-CG, the four pairs that are physically identical, i.e., are the same on both the relevant and irrelevant dimensions. (B) SR-SG, SG-SR, CR-CG, CG-CR, the four pairs that are the same on the relevant dimension of form but differ on the irrelevant dimension of color. (C) SR-CR, CR-SR, SG-CG, CG-SG, the four pairs that differ on the relevant dimension but are the same on the irrelevant dimension. (D) SR-CG, CG-SR, SG-CR, CR-SG, the four pairs that differ on both the relevant and irrelevant dimensions. In the actual experiment, the subject is instructed to respond "same" whenever the two stimuli are the same on the relevant dimension, and to respond "different" whenever the two stimuli are different on the relevant dimension. These instructions mean that the subject is to respond "same" to stimulus groups A and B, and "different" to stimulus groups $C$ and $D$.

Interpretation of results involves comparing the $B$ pairs with the A pairs (same on the relevant dimension), and the $\mathrm{D}$ pairs with the $\mathrm{C}$ pairs (different on the relevant dimension). The first comparison can mostly directly be interpreted in terms of selective attention, since if RT to the B pairs is the same as that to the A pairs, the subject has been able to ignore the difference on the irrelevant dimension, and this result would be indicative of separable dimensions. If RT to the B pairs is greater than that to the A pairs, then the subject has been unable to ignore the irrelevant dimension, and the interpretation would be that the dimensions were integral. Thus the A pairs are in effect the control pairs, and the B pairs the experimental pairs.

The second comparison, between the $\mathrm{C}$ and $\mathrm{D}$ different pairs has often been interpreted in terms of selective attention as well, since if there is a difference in RT between these two pairs, the subject has not been able to ignore the irrelevant dimension. However, it is more logical to consider the comparison between these two groups of pairs in terms of correlated discrimination, since the C pairs are comparable to simple discrimination, with the irrelevant dimension being constant between the members of a pair, and the $\mathrm{D}$ pairs are comparable to correlated discrimination, with the stimuli of a pair differing on both dimensions. Thus, for correlated discrimination, the $\mathrm{C}$ pairs are the controls and the $\mathrm{D}$ pairs the experimental pairs. If the correlated pairs provide a lower RT than do the pairs with one dimension constant, then there has been a gain in discrimination due to the correlation, and the interpretation would be the same as for the classification task.

To summarize, if stimulus dimensions are integral, then RT for B pairs should be higher than for A pairs, and RT for $D$ pairs should be lower than for $C$ pairs, this joint outcome indicating a failure of selective attention and a correlation gain. If stimulus dimensions are separable, then RT for A and B pairs should be the same, and RT for $C$ and $D$ pairs should also be the same, this outcome indicating successful selective attention and no correlation gain. Thus this most elementary use of the comparison task should allow interpretations comparable to those that come from the speeded classification task.

Results with comparison task. Farell (1985, especially pp. 428-430) has recently provided a major review of results with the same-different task, and results pertinent to this use of the comparison task are discussed there. Nickerson's (1972) review is also helpful. The nature of the results can be summarized fairly easily: For same stimulus pairs, when an irrelevant dimension differs in the pair, RT always is greater than when the pair is also the same on the irrelevant dimension, the result indicating a failure of selective attention. As Santee and Egeth (1980) noted, these results have been obtained with dimensions that have been shown to be separable from results from classification tasks. For different stimulus pairs, the results have been very inconsistent, ranging from an increase in RT with irrelevant disparity (e.g., Larsen \& Bundesen, 1978), to a decrease in RT (e.g., Garner, Podgorny, \& Frasca, 1982), to no effect or an inconsistent effect (e.g., Dixon \& Just, 1978).

To some extent this difference between results with same pairs (presumably measuring selective attention) and with different pairs (presumably measuring the correlation effect) are in keeping with results obtained with the classification tasks. With these tasks, interference with the selective attention task is nearly always much greater than facilitation with the correlated discrimination task. Thus effects showing correlation gain are smaller (possibly due to floor effects on RT) than effects showing a failure of selective attention (where no floor effect exists, because RT becomes larger with failure of selective attention).

Control experiments. Yet the differences between the classification and the comparison tasks involve not just different results, but fundamental differences due to the way the stimulus sets are constructed. As outlined here, the simplest way to think of the comparison task is with an experiment using all 16 pairs of stimuli. In the classification tasks, however, either just two or four stimuli are used in a particular experimental task. This difference could account for some of the discrepancy between 
results obtained with the two procedures. In recognition of this fact, several authors have used control experiments in which just two stimuli are used, thus four pairs. Santee and Egeth (1980) used such controls, with the relevant dimension providing the two stimuli, the irrelevant dimension being held constant at one of its levels. Hawkins and Shigley (1972) had used essentially equivalent controls, except that the irrelevant dimension was removed rather than being held constant. To illustrate with the form-color stimuli used earlier, the two stimuli might be SR and CR, with pairs SR-SR and CR-CR as same pairs, and SR-CR and CR-SR as different pairs. With this type of control, the data serve as controls only for the $A$ and $C$ stimulus pairs, that is, those in the full set of 16 pairs that have the irrelevant dimension at a constant level. The results from these control experiments showed that RT for the equivalent stimulus pairs was smaller when the pairs were part of the set of just 4 pairs rather than the full set of 16 pairs, for both same and different pairs. Thus there is the suggestion that the use of a larger set of stimuli (or pairs) influences the outcome.

Smith (1986) argued that Santee and Egeth got their particular results because they failed to use other controls pertinent to the B and D sets of stimulus pairs, in which the irrelevant dimension is different between the two stimuli in a pair. If the difference in the irrelevant dimension is held constant, then there are still just four stimulus pairs generated by combinations of the two levels of the relevant dimension. To illustrate once again with the form-color stimuli, the two same pairs might be CR-CG and SR-SG, and the two different pairs would be CR-SG and SR-CG. The four pairs are generated from the two levels of the relevant dimension, but the irrelevant dimension is always $R$ and $G$, thus different within each pair, but with this difference constant across pairs. These pairs correspond to the B pairs (same on relevant but different on irrelevant dimensions) and the $\mathrm{D}$ pairs (different on both the relevant and irrelevant dimensions). Smith ran such controls and obtained higher RT data from them than with the irrelevant dimension the same across pairs, again for both same and different pairs. Thus the increased number of stimulus pairs in the comparison task may be a factor, but this increased number also produces more complex types of stimulus pairs, and this complexity may be an additional factor in the different results obtained with the comparison tasks and the classification tasks.

Actually, there is still another type of control possible, one that is more relevant to understanding the role of correlation in stimulus comparison. To illustrate with the form-color dimensions that have been used so far, two stimuli might be SR and CG. With these stimuli, the same pairs would be SR-SR and CG-CG, and the different pairs would be SR-CG and CG-SR. With these stimuli, the same pairs are from the A group of pairs, those the same on both the relevant and irrelevant dimensions, but the different pairs are from the $\mathrm{D}$ group of pairs, those differing in both dimensions. Dykes (1979) used such a correlation control, with accuracy measured rather than RT, and obtained the highest accuracy of any of the several conditions he ran with the pairs differing in both dimensions.

Of importance is the fact that none of these various types of control experiment directly controls for the two comparisons that $I$ have argued are parallel to those for the classification tasks. These comparisons are B versus A stimuli for selective attention, and $D$ versus $C$ stimuli for correlation gain. The various controls described always cross these two pairs of stimulus types, being A versus C for Santee and Egeth (1980), B versus D for Smith (1986), and A versus D for Dykes (1979). Furthermore, it is not obvious how such more direct controls can be run. Therefore, in trying to understand the nature of the discrepancy with the two basic tasks, a still different approach seems worthwhile.

Additional stimuli. The approach to be taken in the present comparison experiment has much in common with another line of research in which extra stimuli are added to the two stimuli to be judged in a comparison task. The paper of most direct relevance to this approach is that of Eriksen, O'Hara, and Eriksen (1982), although an earlier paper by Krueger (1973) had used a similar approach. The experimental paradigm is quite simple: Two letters are displayed and are to be judged "same" or "different." On some trials these two letters are all that appear. On other trials, however, an additional "noise" letter is also displayed. The subject is never to respond to the extra letter, but its similarity to one or both of the target letters affects speed of response. With same letter pairs, the more dissimilar the noise (irrelevant) letter is to the letters to be judged, the greater the RT. With different letter pairs, RT is faster when the extraneous letter is different from both of the target letters than when the extraneous letter is identical to one of the target letters.

In interpreted form, the subjects cannot ignore the noise letter with same stimuli, thus exhibiting a failure of selective attention. With different stimuli, the more different the noise letter, the faster the RT. If the greater difference of the added letter is interpreted as providing more correlated information, then the result with different letter pairs demonstrates the positive effect of correlation. Eriksen et al. (1982) interpreted their results in terms of response competition, but the more general term compatibility is preferred for present purposes, since both compatability and incompatibility can occur between the responses required for two or more stimuli or stimulus dimensions. As a result, there is the possibility of both response facilitation and interference, not just interference resulting from competition.

\section{THE COMPARISON EXPERIMENT}

The present comparison experiment took an approach similar to that of the addition of an irrelevant letter, except that an irrelevant, redundant dimension was added to a set of stimuli, rather than a third stimulus to the pair to be judged. The primary, and important, advantage of 
this procedure is that the number of stimulus pairs to be judged does not have to be changed. Thus the problems involved in using various control experiments, always with a smaller set of pairs, is avoided. In the usual comparison experiment concerned with selective attention, when an additional irrelevant dimension is added to a set of stimuli, it is crossed with the existing dimensions, thus producing a larger set of stimuli and stimulus pairs. This confound is avoided in the present use of an added redundant dimension, while still allowing for determination of the effect of a redundant dimension.

The experimental design is outlined in Table 1. The basic experiment with black letters is the control condition. Four letters, C, O, L, and T, are the four stimuli that form pairs for the comparison task. These letters have been used before (e.g., Garner, 1987) because they provide two different levels of discriminability between letter pairs. The letters that share curvature or straightness ( $C$ and $\mathrm{O}, \mathrm{L}$ and $\mathrm{T}$ ) are difficult to discriminate, while the other four pairs of letters are easier to discriminate because use can be made of the curved-straight distinction. In the same-different task, these four letters form 16 pairs, but these pairs can be divided into four different groups of different types. Same pairs include C-C, $\mathrm{O}-\mathrm{O}, \mathrm{L}-\mathrm{L}$, and T-T. Hard different pairs are $\mathrm{C}-\mathrm{O}, \mathrm{O}-\mathrm{C}$, $\mathrm{L}-\mathrm{T}$, and $\mathrm{T}-\mathrm{L}$. There are two groups of easy different pairs, arbitrarily labeled Easy 1 and Easy 2, but the differences between these two groups are useful in the experimental manipulation. As labeled in Table 1, Easy 1 different pairs are $\mathrm{C}-\mathrm{L}, \mathrm{L}-\mathrm{C}, \mathrm{O}-\mathrm{T}$, and $\mathrm{T}-\mathrm{O}$; Easy 2 different pairs are $\mathrm{C}-\mathrm{T}, \mathrm{T}-\mathrm{C}, \mathrm{O}-\mathrm{L}$, and $\mathrm{L}-\mathrm{O}$.

The use of these four groups allows the usual comparison between RTs for same and different pairs, but it also allows comparison between hard and easy different pairs. Note that this design does not include stimulus pairs in which the relevant dimension is the same, but the irrelevant dimension is different.

\section{Experimental Conditions}

The letters were always black in the control condition, as shown in Table 1. Color was used as the additional, redundant dimension in each of two experimental conditions labeled Color 1 and Color 2 . In each of these conditions, two of the letters were red and two were green, $\mathrm{C}$ and $\mathrm{O}$ being red and $\mathrm{L}$ and $\mathrm{T}$ being green for Color 1 , and $\mathrm{C}$ and $\mathrm{L}$ being red and $\mathrm{O}$ and $\mathrm{T}$ being green for Color 2. Note that color was not crossed with letters, a procedure that would have produced eight different stimuli, and 64 stimulus pairs. For a particular condition, each letter had the same color throughout all trials of that condition; there was no irrelevant variation of color with respect to letter, and thus color was simply redundant.

This procedure means that all same letter pairs had the same color as well, so that color was compatible with the required "same" response for all such pairs. This procedure was used because the primary interest was in understanding what happens with the different pairs. The literature is not confusing regarding same pairs, so the
Table 1

Color Assignments in the Design of the Comparison Experiment

\begin{tabular}{|c|c|c|c|c|}
\hline \multirow[b]{2}{*}{ Pair Type } & \multirow{2}{*}{$\begin{array}{l}\text { Letter } \\
\text { Pair }\end{array}$} & \multicolumn{3}{|c|}{ Condition } \\
\hline & & Control & Color 1 & Color 2 \\
\hline Same & $\begin{array}{l}\mathrm{C}-\mathrm{C} \\
\mathrm{O}-\mathrm{O} \\
\mathrm{L}-\mathrm{L} \\
\mathrm{T}-\mathrm{T}\end{array}$ & $\begin{array}{l}\text { B-B } \\
\text { B-B } \\
\text { B-B } \\
\text { B-B }\end{array}$ & $\begin{array}{c}\text { R-R } \\
\text { R-R } \\
\text { G-G } \\
\text { G-G } \\
\text { (Compatible) }\end{array}$ & $\begin{array}{c}\text { R-R } \\
\text { G-G } \\
\text { R-R } \\
\text { G-G } \\
\text { (Compatible) }\end{array}$ \\
\hline $\begin{array}{l}\text { Hard } \\
\text { Different }\end{array}$ & $\begin{array}{l}\mathrm{C}-\mathrm{O} \\
\mathrm{O}-\mathrm{C} \\
\mathrm{L}-\mathrm{T} \\
\mathrm{T}-\mathrm{L}\end{array}$ & $\begin{array}{l}\text { B-B } \\
\text { B-B } \\
\text { B-B } \\
\text { B-B }\end{array}$ & $\begin{array}{c}\text { R-R } \\
\text { R-R } \\
\text { G-G } \\
\text { G-G } \\
\text { (Incompatible) }\end{array}$ & $\begin{array}{c}\text { R-G } \\
\text { G-R } \\
\text { R-G } \\
\text { G-R } \\
\text { (Compatible) }\end{array}$ \\
\hline $\begin{array}{l}\text { Easy } 1 \\
\text { Different }\end{array}$ & $\begin{array}{l}\text { C-L } \\
\text { L-C } \\
\text { O-T } \\
\text { T-O }\end{array}$ & $\begin{array}{l}\text { B-B } \\
\text { B-B } \\
\text { B-B } \\
\text { B-B }\end{array}$ & $\begin{array}{c}\text { R-G } \\
\text { G-R } \\
\text { R-G } \\
\text { G-R } \\
\text { (Compatible) }\end{array}$ & $\begin{array}{c}\text { R-R } \\
\mathbf{R}-\mathbf{R} \\
\mathbf{G}-\mathbf{G} \\
\mathbf{G}-\mathbf{G} \\
\text { (Incompatible) }\end{array}$ \\
\hline $\begin{array}{l}\text { Easy } 2 \\
\text { Different }\end{array}$ & $\begin{array}{l}\text { C-T } \\
\text { T-C } \\
\text { O-L } \\
\text { L-O }\end{array}$ & $\begin{array}{l}\text { B-B } \\
\text { B-B } \\
\text { B-B } \\
\text { B-B }\end{array}$ & $\begin{array}{c}\text { R-G } \\
\text { G-R } \\
\mathbf{R}-\mathbf{G} \\
\mathbf{G}-\mathbf{R} \\
\text { (Compatible) }\end{array}$ & $\begin{array}{c}\text { R-G } \\
\text { G-R } \\
\text { G-R } \\
\text { R-G } \\
\text { (Compatible) }\end{array}$ \\
\hline
\end{tabular}

Note-Compatibility refers to the same-different relaton of the letters and the colors. $\mathbf{B}=$ black, $\mathbf{R}=$ red, $\mathbf{G}=$ green.

fact that all conditions produced compatible colors and letters for same pairs was an acceptable loss. However, the different color assignments for Color 1 and Color 2 allowed different compatible or incompatible relations for the various types of different pairs. Specifically, Color 1 produced letter pairs that had the same colors for hard different letters (thus providing an incompatible relation between letters and color); it produced letter pairs with different colors for both Easy 1 and Easy 2 different letters. Color 2 changed these relations: It produced letter pairs that had different colors for hard different letters (thus providing a compatible relation between letters and colors); and it produced letter pairs with the same color for Easy 1 different pairs (thus providing an incompatible relation between letters and color). Easy 2 different letters maintained a compatible relation with Color 2 . A third assignment of colors to letters could have produced a reversal in compatibility between Easy 1 and Easy 2, but since these two letter groups shared the same properties, there was no need for such a condition.

The properties of this experimental arrangement can be reviewed by looking at the four groups of letter pairs and seeing how the experimental conditions changed the compatibility relations between letters and colors. For the control condition, all letters were black, so the compatibility question is irrelevant. But for same pairs, both Color 1 and Color 2 provided color-letter compatibility. For hard different pairs, Color 1 provided an incompatible color-letter relation, and Color 2 provided a compatible relation. For Easy 1 different pairs, these relations were reversed: Color 1 provided color-letter compatibility and Color 2 provided color-letter incompatibility. For Easy 2 
different pairs, both experimental conditions provided a compatible color-letter relation. Thus this group of letter pairs provided a control for the effects of compatible color-letter relations on the other two groups of different letter pairs, since there should be no difference between the Color 1 and Color 2 conditions.

\section{Purpose of Experiment}

This experimental arrangement was intended to provide information about the effect of a redundant dimension on discrimination speed with a stimulus comparison task. Since previous results have consistently shown a failure of selective attention on same stimulus pairs, the design was focused on the nature of the different stimulus pairs. Two primary factors were investigated: first, the effect of compatibility of the redundant dimension with the primary dimension of letters; second, the role of stimulus discriminability in determining whether compatibility had an effect on speed of discrimination. In other words, is there an interaction between the effects of discriminability and the effects of compatibility? A design was used which allowed differences in discriminability and in compatibility to occur with a fixed number of stimulus pairs, to avoid the confound of an increased size of stimulus set when irrelevant dimensions were crossed with relevant dimensions.

Since the discrepancies previously found between results with speeded classification and comparison tasks have concerned separable dimensions, the dimensions of form (the letters) and color were used, since these are generally considered to be separable dimensions (Gottwald \& Garner, 1972).

\section{Method}

Subjects. Eighteen subjects, recruited from Yale students and staff, were paid for participation. All had normal or corrected vision, and before being used as subjects all were given a simple color test in which they were required to select a good green and a good red from a random array of colors. All subjects were able to pass this test.

Stimuli. The stimuli for all conditions were the uppercase letters C, O, L, and T, presented in a Scientific Prototype tachistoscope, for which $12.7 \times 17.8 \mathrm{~cm}$ cards were used to display the stimuli. The actual stimuli used were Letraset 725, 30-pt Helvetica Medium. These particular letters were used because two of them are composed of only curved lines and the other two of only straight lines, with no serifs or other flourishes that would prevent a clear distinction between curved and straight letters. The actual physical measurements of the $O$ (the widest letter) were $8.0 \mathrm{~mm}$ high and $7.5 \mathrm{~mm}$ wide, with a line thickness of $1.6 \mathrm{~mm}$. The stimuli were viewed at a distance of $127 \mathrm{~cm}$, at which the visual angle of the height was $.36^{\circ}$. The background luminance was $17.1 \mathrm{~cd} / \mathrm{m}^{2}$.

Stimulus presentation was simultaneous, with two letters on a stimulus card. The two letters were presented in the lower left and upper right quadrants of the stimulus field, off the diagonal, in order to prevent special properties of the pair, such as symmetries, from influencing RTs. The bottom of the top letter and the top of the bottom letter were at the horizontal midline. The letters were also centered $8 \mathrm{~mm}$ to the right or left of the vertical midline. This arrangement means that the center of each stimulus was $.36^{\circ}$ to the right or left of center and $.18^{\circ}$ above or below center.

When the letters were colored, they were either red (approximate Munsell equivalent SR 4/14) or green (approximate Munsell equivalent $2.5 G 4 / 8$ ). The letters were black for the control condition.

Task. The task was a conventional simultaneous same-different task. Two stimuli were presented, and the subject responded "same" or "different" by pushing a switch lever, located under the tachistoscope, up or down with the right hand. At all times when the stimuli were not on, a reminder card was present, with a fixation dot in the center and the response assignment indicated by the words "same" or "different" at the top or bottom of the card. Response assignments were counterbalanced across subjects. The task was always defined in terms of sameness or difference of the letters, and at no time was the subject instructed to respond (or even attend) to color in the conditions in which the letters were colored.

Trials. The subject initiated a trial by pushing a button switch held in the left hand. Immediately the reminder field was replaced by the stimulus field, which remained on for $1,000 \mathrm{msec}$, during which time the subject responded. Then the reminder card came on again. The experimenter put in a new stimulus card, the sound of which informed the subject that it was possible to start a new trial. A computer randomized the order of stimulus presentations, and recorded the response and the RT for each trial.

Sessions. Each subject participated for three sessions of about $1 \mathrm{~h}$ each. A single condition was used for each session. The session started with 50 practice trials for that condition, followed by 384 real trials, 24 for each of the 16 pairs. Note that this arrangement means that only $25 \%$ of the trials were same trials.

Experimental design. All of the six possible sequences in which three conditions can be run were used. Three subjects were assigned to each of the six sequences. The use of as many as $\mathbf{5 0}$ practice trials at the beginning of each session was expected to eliminate or at least minimize the effect of order within each sequence, but it failed to eliminate it completely, there being a 29 msec difference between the first and the third sessions, averaged over all subjects. A correction for this effect was used as noted below.

Data analysis. Errors for the three conditions ranged from $2.3 \%$ to $2.7 \%$. Errors for the four types of letter pairs, averaged across the conditions, were $6.5 \%, 2.1 \%$, and $0.6 \%$ for same, hard differ$e n t$, and the two combined easy pairs, respectively. Since this ordering corresponds exactly with the obtained RT data, and the errors are too few for useful analysis, all further analyses use RT data only.

For each session and each pair of stimuli, a median RT for correct trials was calculated for each subject, the median being used to minimize the effect of outliers. All further analyses were based on means of these medians. Because of the 29-msec order effect, all median scores were corrected to eliminate the effect. The correction was based on the single mean score for order based on all conditions and subjects. Since this correction was applied to all data, it produces no change in mean scores. Furthermore, it produces no change in any analyses between stimulus types within a condition. However, it reduces the error variance in analyses done between conditions for a given pair type. This procedure is equivalent to using a Latin square residual as an error term, but since it was desired to do analyses between conditions for each letter pair type separately, the single correction applied to all data in a condition is more conservative than treating each analysis for a pair type as though the data had been obtained in separate experiments.

These corrected scores were subjected to various analyses of variance (ANOVAs), and various contrast tests were used for specific questions (Winer, 1971, p. 170ff). For all statistical tests, a significance level of $p<.01$ was used unless otherwise noted.

\section{Results}

The data for this experiment, by condition and type of letter pair, are displayed in Table 2 . The basic pattern of data is quite straightforward. Same pairs produced the highest RTs. The hard different letter pairs were more 
Table 2

Mean Reaction Times (in msec) for Conditions and Types of Letter Pairs in the Comparison Experiment

\begin{tabular}{lcll}
\hline & \multicolumn{3}{c}{ Condition } \\
\cline { 2 - 4 } \multicolumn{1}{c}{ Pair Type } & Control & Color 1 & Color 2 \\
\hline Same & 473 & 486 (C) & $473(\mathrm{C})$ \\
Hard Different & 420 & $454(\mathrm{I})$ & 389 (C) \\
Easy 1 Different & 373 & $377(\mathrm{C})$ & 393 (I) \\
Easy 2 Different & 374 & $379(\mathrm{C})$ & 374 (C) \\
\hline
\end{tabular}

Note-(C) and (I) refer to compatible and incompatible relations between letters and colors as shown in Table 1.

difficult than the easy different pairs in the control condition, and RT for the hard different pairs became higher when color was incompatible and lower when color was compatible. Thus both interference and facilitation occurred as a result of changes in color-letter compatibility with this pair type. The Easy 1 different pairs were faster than the hard different pairs in the control condition, remained the same in the Color 1 condition when color was compatible, and increased in RT when color was incompatible. Thus only interference occurred with this pair type. The Easy 2 different pairs were as fast as the Easy 1 pairs in the control condition, and did not change with the two color conditions, in both of which color was compatible. With respect to different stimulus pairs only, both facilitation and interference (depending on compatibility) occurred when color was introduced as a redundant dimension and the letters were hard to discriminate; when the letters were easy to discriminate, only interference occurred when color was incompatible with the letter pair.

An overall ANOVA of these data is of little value, since the primary interest is in the specific changes that occurred when color and letters were compatible or incompatible. To establish the significance of these effects, a series of ANOVAs was carried out, both between pair types within conditions and between conditions within pair types.

Same pairs. Same pairs were not of particular importance in this experiment, as the design of the experiment and the results show. The differences between RTs for same pairs between conditions were not significant overall, nor was the $13 \mathrm{msec}$ greater RT for the Color 1 condition significantly different from either of the other two conditions in single contrasts. On the other hand, RT for same pairs was significantly higher than the highest RT for any type of different pair for each condition $[F \mathrm{~s}(1,51)$ $=80.26,21.16$, and 299.16 , respectively, for control, Color 1, and Color 2 conditions]. This greater RT for same pairs is almost certainly due simply to the fact that same pairs occurred only $25 \%$ of the time. The fact that there was no difference in same-pair RTs for the different conditions affirms that the introduction of the redundant color had no overall deleterious effect on performance. Further analyses do not use data for same pairs, but only data for different pairs.

Different pairs. When different RTs are examined across types of different pairs within each condition, the following picture emerges: In the control condition, RT for hard different pairs was nearly $50 \mathrm{msec}$ greater than that for either of the two easy different pairs $[F \mathrm{~s}(1,34)$ $=118.85$ and 112.62 for Easy 1 and Easy 2, respectively], and these were not different from each other. The strength of this result is also shown by the fact that all 18 subjects showed it. This result confirms the fact that $\mathrm{C}-\mathrm{O}$ and $\mathrm{L}-\mathrm{T}$ pairs are in fact more difficult to discriminate than are the other pairs, as was intended. In the Color 1 condition, RT for hard different pairs was again greater (by 75 and $77 \mathrm{msec}$ ) than either of the easy pairs $[F \mathrm{~s}(1,34)=161.25$ and 152.19 for Easy 1 and Easy 2$]$, and these were not different from each other. Once again, the effect was shown by all 18 subjects. In the Color 2 condition, RTs for the hard and Easy 1 different pairs were not different, but each was greater than that for Easy 2 different pairs $\left[F_{\mathrm{S}}(1,34)=17.06\right.$ and 29.26 , respectively]. This result was shown by 16 of the 18 subjects. Thus the Color 2 condition, which produced compatible color for the hard different pairs and incompatible color for the Easy 1 different pairs, made these two types of pairs equally difficult, and both more difficult than the Easy 2 pairs with compatible color.

When different RTs are examined across conditions within each pair type, the following picture emerges: For hard different pairs, Color 1 significantly increased RT by $34 \mathrm{msec}$ over the RT for the control condition [ $F(1$, $34)=11.48$ ], and Color 2 decreased RT by $31 \mathrm{msec}$ $[F(1,34)=9.69]$. Thus incompatible color increased $\mathrm{RT}$ and compatible color decreased RT. For Easy 1 different pairs, there was no difference between RT for the control condition and that for Color 1 , with compatible color. With Color 2, however, RT was increased over the control condition by $20 \mathrm{msec}[F(1,34)=8.80]$, and this effect was shown by 16 of the 18 subjects. RT for Color 2 was also $16 \mathrm{msec}$ greater than that for Color $1[F(1,34)$ $=5.67, p<.025]$. For Easy 2 different pairs, in which both color conditions produced compatible color, there were no differences between the control and either of the color conditions. Thus, as with same pairs, this result simply confirms that the introduction of color per se did not adversely affect RT.

\section{Discussion}

These results confirm previous results by showing that stimulus dimensions ordinarily considered separable on the basis of speeded classification tasks act like integral dimensions with the comparison task. The particular experimental design used in this experiment was intended to maximize information about the "different" response at the cost of any further information about the "same" response, and it was also intended to provide an experimental paradigm in which the number of stimulus pairs was the same for both the control and the experimental conditions. These intentions were effective.

For different pairs of letters, when color was added as a totally redundant dimension that did not increase the number of pairs, RT was strongly affected. The effect was 
a joint function of the difficulty of discrimination of the letter pairs and of the compatibility of the colors and letters. "Compatibility," of course, is used in the sense that if a response to color had ever been required, it would have been the same as or different from that required for the letters. Since our only concern is with different letter pairs, when colors were the same they were incompatible, and when they were different they were compatible.

With both hard and easy letter pairs, when color was incompatible with the different letters, RT was significantly increased. However, when color was compatible with the different letters, RT was decreased only for the hard different pairs. The actual value obtained in this condition $(389 \mathrm{msec})$ is so close to the values obtained with easy different pairs (lowest value obtained, $373 \mathrm{msec}$ ), that it seems very possible that a floor effect was operating with the easy different letter pairs, so that no improvement was possible with these pairs. Certainly the Color 1 condition, in which the Easy 1 different pairs had compatible color, suggests a complete floor effect, since the RT obtained was $377 \mathrm{msec}, 4 \mathrm{msec}$ higher than the value obtained for the appropriate control condition.

Despite such probable floor effects, these results show that both facilitation and interference can be obtained with different stimulus pairs when a redundant dimension is added that in no way alters the basic control task. Thus the parallel of the results to those obtained with integral dimensions as measured by the speeded classification tasks is even greater than has been obtained before, since both facilitation and interference in speeded classification are defining experimental outcomes for integral dimensions. Furthermore, in the Eriksen et al. (1982) experimental paradigm, some of whose characteristics were used in the present experimental design, facilitation has never been found with the introduction of an additional noise letter, presumably because the additional letter provides enough distraction to raise overall $\mathrm{RT}$ and thus prevent the showing of any possible facilitative effects. But in the present experiment, with the addition of a dimension rather than a stimulus, such facilitation was clearly evident.

\section{THE SPEEDED CLASSIFICATION EXPERIMENTS}

This entire comparison experiment and the interpretation of its results are based on the assumption that color and form are separable dimensions. However, these particular forms are not the squares, triangles, and so forth, usually used in such experiments; furthermore, since the color appeared only in the letter itself and was therefore narrow, it is possible that these particular letters and particular color presentations are not separable. Therefore, two additional experiments were run with the full array of speeded classification tasks, to determine whether these zolors and these forms were in fact separable, or, if not, whether color was at least separable from form, since that 's all that is required. Such a result could occur if the colors and letters had different discriminabilities, since integrality becomes asymmetric under such circumstances (Gamer \& Felfoldy, 1970; Felfoldy \& Garner, 1971).

The two experiments each used two letters and the same two colors as those used in the comparison experiment. One experiment used the letters $\mathrm{O}$ and $\mathrm{C}$ (a hard pair to discriminate) and the other experiment used the letters $O$ and $T$ (an easy pair to discriminate). In all other respects the two experiments were identical.

\section{Method}

Subjects. Thirty-two subjects, recruited from Yale students and staff, were paid for participation. Sixteen were used in each experiment. None had been used in the comparison experiment. All had normal or corrected vision, and before being used were given the same simple color test used in the comparison experiment. All the subjects were able to pass this test.

Stimuli. There were four stimuli in each experiment, a red $C$, a red $\mathrm{O}$, a green $\mathrm{C}$, and a green $\mathrm{O}$ for the $\mathrm{O}-\mathrm{C}$ experiment, and the equivalents for the $\mathrm{O}-\mathrm{T}$ experiment. The actual letter stimuli were identical to those used in the comparison experiment, as was background illumination. The stimuli were presented one per trial, centered in the visual field.

Tasks. Eight different tasks were used in each experiment, of three different types. Four tasks required simple discrimination of a single dimension, either letter or color. For each dimension, two tasks were used, one for each level of the dimension held constant. Thus there were two color discrimination tasks and two letter discrimination tasks. Two tasks required correlated discrimination. The two ways of correlating the dimensions were used, for example, red $C$ versus green $O$, or green $C$ versus red $O$. Thus there were six discrimination tasks in all. The last two tasks were both full classifications in which all four stimuli were used, and classification was required by letter or by color, with the other dimension varying irrelevantly.

At all times between actual trials, a reminder card was present. It indicated the appropriate up or down response with the switch lever located under the tachistoscope. The actual letter(s) were shown at the top and bottom of the cards to indicate the direction of the response. The reminder card also had a fixation dot in the center. Response assignments were counterbalanced across subjects.

Trials. The subject initiated a trial by pushing a button switch held in the left hand. The reminder card remained on for $400 \mathrm{msec}$, after which the field went blank for $100 \mathrm{msec}$. Then the stimulus came on for 1,000 msec, during which time the subject responded. (This change in procedure from that of the comparison task was made because the letter appeared in the middle of the field at the same location as the fixation dot, and the blank interval before the stimulus was used to prevent possible interference.) Other aspects of trial procedure were the same as in the comparison experiment.

Sessions. Each subject participated for two sessions of about $1 \mathrm{~h}$ each. All eight tasks were used in each session. Practice trials were given before each new task, 10 for each of the first four tasks, then five. For the real trials, there were 25 trials per stimulus per task. Thus the six discrimination tasks, with just two alternative stimuli, had a total of 50 trials, and the two classification tasks had a total of 100 trials. All data from the first session were considered practice and were not analyzed; data presented are from the second session only.

Experimental design. The eight tasks were arranged in a Latin square for 8 subjects. The Latin square was constrained so that each subject got one classification task in each half of the eight and no subject got the two correlated tasks consecutively. The same Latin square was reversed for a second group of 8 subjects. The same experimental design was used for both the O-C and the O-T experiments. 
Data analysis. Errors averaged $2.5 \%$ and $2.6 \%$ for the $\mathrm{O}-\mathrm{C}$ and $\mathrm{O}-\mathrm{T}$ experiments, respectively. Correlation between error percentages and conditions was -.05 within the $\mathrm{O}-\mathrm{C}$ experiment and .55 within the $\mathrm{O}-\mathrm{T}$ experiment. Thus error rates are too low to be useful and there is no evidence of a speed-accuracy tradeoff, so all further analyses use RT data only.

For each subject, for each letter in each task, a median RT was calculated for correct trials. All further analyses were based on means of these medians. Furthermore, within each experiment, the data for each pair of single dimension and correlated discrimination trials were averaged, since there was no evidence of any consistent differences between the two tasks involving each pair. Thus further data analyses were done with two simple discrimination tasks for single dimensions (one for each dimension), one correlated discrimination task, and two full classification tasks. Combining the data for the various discrimination tasks, in which there were $\mathbf{5 0}$ trials, gives a total number of trials of 100 , the same as that used for the classification tasks. Therefore the results as shown are based on equal numbers of trials for a total of five tasks. Other aspects of data analysis were the same as for the comparison experiment.

\section{Results}

The data for the five tasks for both experiments are displayed in Table 3. With one exception, the overall picture obtained is the same for both experiments.

O-C experiment. Letter discrimination was faster than color discrimination, but by a nonsignificant $7 \mathrm{msec}$, with only 11 of the 16 subjects showing this effect. Correlated discrimination was faster than the faster single dimension (letter) by a nonsignificant $5 \mathrm{msec}$, with 8.5 of the 16 subjects being faster on the correlated pairs than on each of the two single-dimension pairs. Thus there is no evidence of a gain due to the use of correlated dimensions. However, the slightly better performance with letters than with color showed up more strongly in the classification tasks, with letter classification being $19 \mathrm{msec}$ faster than color classification $[F(1,60)=19.64]$. Letter classification was only $1 \mathrm{msec}$ slower than letter discrimination, so there is clear evidence of selective attention to letters when color varied irrelevantly. On the other hand, color classification was significantly slower by $13 \mathrm{msec}$ than its control discrimination $[F(1,60)=10.08]$. Thus random variation of letter interfered with color classification, but not conversely.

O-T experiment. The O-T letters were chosen because they are more easily discriminated than the $\mathrm{O}-\mathrm{C}$ letters. The effect of this change is clear in the results. Letter discrimination was $35 \mathrm{msec}$ faster than color discrimination $[F(1,60)=34.51]$, and this effect was true for all 16 subjects. Correlated discrimination was actually slightly slower (by a nonsignificant $8 \mathrm{msec}$ ) than the faster letter

Table 3

Mean Reaction Times (in msec) for the Five Tasks in the Two Speeded Classification Experiments

\begin{tabular}{ccccccc}
\hline & \multicolumn{4}{c}{ Task } \\
\cline { 2 - 5 } & \multicolumn{3}{c}{ Discrimination } & & \multicolumn{2}{c}{ Classification } \\
\cline { 2 - 4 } \cline { 6 - 7 } Experiment & Color & Letter & Correlated & & Color & Letter \\
\hline O-C & 391 & 384 & 379 & & 404 & 385 \\
O-T & 406 & 371 & 379 & & 419 & 389 \\
\hline
\end{tabular}

discrimination, with only 4 of the subjects showing a lower RT with correlated discrimination. Thus again there is no evidence of a correlation gain. The greater discriminability of letters was also evident in a comparison of the two classification tasks, with letter classification being $30 \mathrm{msec}$ faster than color classification $[F(1,60)=23.29]$. Letter classification was a significant $18 \mathrm{msec}$ slower than letter discrimination $[F(1,60)=9.73]$, although the effect was shown by only 11 of the 16 subjects. So the complete selective attention for letters found in the O-C experiment was not found in the $\mathrm{O}-\mathrm{T}$ experiment. Color classification was a significant $13 \mathrm{msec}$ slower than color discrimination $[F(1,60)=4.28, p<.05]$, and this effect was also shown by only 11 of the 16 subjects. Thus a failure of selective attention was found for both letter and color classification. It should be noted, however, that the effects were not large, and were shown by only 11 of the 16 subjects, a number that is not significantly different from chance. The effects due to dimension were considerably greater than the effects due to a failure of selective attention within each dimension.

\section{Discussion}

The purpose of these two classification experiments was to determine whether the dimensions of color and form as specifically used in these experiments were separable, or, if not, whether color interfered with or facilitated letter discrimination.

The results for the $\mathrm{O}-\mathrm{C}$ letters (the hard ones to discriminate) are very clear. There was no correlation gain, and irrelevant color did not interfere with letter classification, although irrelevant letters did interfere with color classification. Since color was the added redundant dimension in the comparison experiment, this result with the classification procedure is different from that obtained with the comparison procedure. For the hard different letters, there was both facilitation and interference in the comparison task, when color was added as a fixed redundant dimension so as to produce compatibility and incompatibility with the letter comparison task. It should be noted that the failure of color to lower RT when used as a correlated dimension in the speeded classification tasks might have been due to a floor effect, but even so, the inability of irrelevant color variation to prevent selective attention to letters was not.

The results with the O-T letters (the easy ones to discriminate) essentially duplicate those for the $\mathrm{O}-\mathrm{C}$ experiment, with one exception: the fact that irrelevant color variation did interfere with letter classification. Since color is the less discriminable dimension, this result is surprising. It most probably is due to the substantial difference in the configuration of the $\mathrm{O}$ and $\mathrm{T}$ forms, a difference that may have made it difficult to ignore irrelevant variation in another dimension. Even though it is easier to discriminate $\mathrm{O}$ from $\mathrm{T}$ than from $\mathrm{C}$, the $\mathrm{O}-\mathrm{C}$ task allows the subject to use the same basic expectation about location of the stimulus components, a strategy not possible with $\mathrm{O}$ and $\mathrm{T}$. 
Despite this one minor anomaly in the results of the $\mathrm{O}-\mathrm{T}$ experiment, it is clear that color is less discriminable than letters (although marginally so for the $\mathrm{O}-\mathrm{C}$ discrimination), so that its addition as a fixed redundant dimension in the comparison experiment could be expected to be minimal. Yet the effects of incompatible color in producing interference were clear for both easy and hard different pairs; and the effect of compatible color in producing facilitation was equally strong for the hard different pairs.

\section{GENERAL DISCUSSION}

In brief, the results of this comparison experiment confirm and extend the results of earlier experiments that have shown that stimulus dimensions that are essentially separable in classification tasks give results in the comparison task that suggest an integral relation. The comparison task used in the present experiment was designed to allow a redundant dimension to be added to the control condition without altering the number of possible stimuli or the basic task. While this procedure alleviates some difficulties with previous procedures, the results confirm previous results.

\section{Response Compatibility}

The basic question is, why does this difference in outcomes due to task differences exist? The explanation would seem to be due to the most obvious and blatant difference between the two types of task, namely, the nature of the response. Eriksen et al. (1982), in explaining their results with a noise letter in addition to the two letters to be judged "same"' or "different,"' suggested that response competition occurred with the extra letter. In the present comparison experiment, since both facilitation and interference occurred with the addition of color, response compatibility would be the more appropriate and general term to refer to this response interaction.

Simply stated, the dimensions ordinarily used to study the integrality-separability question do not have implicit or explicit responses that are inherently compatible or incompatible. In the present experiments, there is no natural analogic relation between responses to colors and responses to letters. Yet when these same dimensions are combined in a comparison task, response compatibility is immediately produced, since now both dimensions can be either same or different. Thus it would seem most reasonable to explain the differences obtained from the two experimental paradigms as being due to this fundamental change in the nature of the response, a change that introduces compatibility (or incompatibility) between dimensions when none had existed before.

\section{Compatibility in Classification}

Garner $(1976,1983)$ has noted that response interference can exist even with classification tasks, and has argued that when dimensions have natural responses for different levels that can be compatible or incompatible, the dimensions are not really appropriate for studying the nature of integrality. The Stroop phenomenon is one wellknown example, in which color names and the colors the names are printed in can be compatible or incompatible (see Dyer, 1973, for a review of this literature). Two other examples will suffice. Clark and Brownell (1976) used arrows that pointed up or down and were located in an up or down position. The critical thing about such experiments is that the correlated discrimination tasks have to be specified as having a positive or negative correlation, since the same responses can be used for both stimulus dimensions. Clark and Brownell found a much higher RT when the two dimensions were negatively correlated than when they were positively correlated. Redding and Tharp (1981) used lines that could be tilted right or left and that could also be located right or left, thus having compatibility inherent in the responses. Again, with the correlated discrimination tasks, positively correlated dimensions produced a lower RT than negatively correlated dimensions. Incidentally, in each of these examples the positively correlated dimensions actually produced a lower RT than the single-dimensional controls. Thus, as in the comparison task, with correlated discrimination, facilitation occurred when the stimulus dimensions were compatible (positively correlated), and interference occurred when they were incompatible (negatively correlated).

In these examples it is obvious that the two dimensions involved share a common verbal description; thus there is no reason to be surprised when compatibility effects occur. However, many stimulus dimensions may have less obvious, but nevertheless real, compatibilities due to inherent properties of the dimensions or perhaps to learning of equivalences. Therefore in classification experiments it is best if both possible pairings of correlated dimensions be used, rather than a single arbitrarily chosen pairing, which Garner and Felfoldy (1970) used in the original classification experiments.

\section{Conclusion}

In conclusion, the role of response compatibility seems to be crucial in both classification and comparison tasks. When the issue of integrality is studied with classification procedures, stimulus dimensions that have potential response compatibility are usually not used. However, when such dimensions are used, both interference and facilitation occur. When the comparison procedure is used, the problem of compatibility is inherent in the method and cannot be avoided. Thus the different apparent outcomes concerning integrality and separability with the classification and comparison procedures may be readily explained as being due to the fact that the classification procedure does not introduce compatibility when none is inherent in the dimensions, whereas the comparison procedure has a necessary compatibility relation due to the fact that any pair of stimulus dimensions can share the "same" or the "different" response.

The results obtained with the comparison task appear on the surface to imply a failure of selective attention, or more generally an integral relation between the dimensions. Yet two dimensions capable of being processed in parallel, but with only one of them relevant, could give a result showing selective attention with the classification 
procedure, since only one response system of the two must be used. But in the comparison task, both dimensions have to use the same response system, and thus interference or facilitation can occur. Because of this compatibility issue, no clear interpretation concerning selective attention is possible with the comparison procedure. Integral and separable dimensions would both give the same pattern of results. Thus, once again, it seems necessary to say that the role of response compatibility is crucial in both classification and comparison tasks.

\section{REFERENCES}

Clark, H. H., \& Brownele, H. H. (1976). Position, direction, and their perceptual integrality. Perception \& Psychophysics, 19, 328-334.

Dixon, P., JUST, M. A. (1978). Normalization of irrelevant dimensions in stimulus comparisons. Joumal of Experimental Psychology: Human Perception \& Performance, 4, 36-46.

DYER, F. N. (1973). The Stroop phenomenon and its use in the study of perceptual, cognitive, and response processes. Memory \& Cognition, 1, 106-120.

DYKES, J. R., JR. (1979). A demonstration of selection of analyzers for integral dimensions. Journal of Experimental Psychology: Human Perception \& Performance, 5, 734-745.

Eriksen, C. W., O'Hara, W. P., \& Eruksen, B. (1982). Response competition effects in same-different judgments. Perception \& Psychophysics, 32, 261-270.

FARELL, B. (1985). "Same"-“"different"” judgments: A review of current controversies in perceptual comparisons. Psychological Bulletin, 98, 419-456.

Felfoldy, G. L., \& GARNer, W. R. (1971). The effects on speeded classification of implicit and explicit instructions regarding redundant dimensions. Perception \& Psychophysics, 9, 289-292.

GARNER, W. R. (1970). The stimulus in information processing. American Psychologist, 25, 350-358.

GARNER, W. R. (1974). The processing of information and structure. Potomac, MD: Erlbaum.

GARNER, W. R. (1976). Interaction of stimulus dimensions in concept and choice processes. Cognitive Psychology, 8, 98-123.
GARNER, W. R. (1983). Asymmetric interactions of stimulus dimensions in perceptual information processing. In T. J. Tighe \& B. E. Shepp (Eds.), Perception, cognition, and development: Interactional analyses (pp. 1-38). Hillsdale, NJ: Erlbaum.

GARNER, W. R. (1987). Location and color as cuing dimensions in contingent classification. Perception \& Psychophysics, 41, 202-210.

GARNER, W. R., Felfoldy, G. L. (1970). Integrality of stimulus dimensions in various types of information processing. Cognitive Psychology, 1, 225-241.

Garner, W. R., Podgorny, P., \& Frasca, E. M. (1982). Physical and cognitive dimensions in stimulus comparison. Perception \& Psychophysics, 31, 507-522.

GoTTWALD, R. L., G GARNER, W. R. (1972). Effects of focusing strategy on speeded classification with grouping, filtering, and condensation tasks. Perception \& Psychophysics, 11, 179-182.

Hawkins, H. L., Shigley, R. H. (1972). Irrelevant information and processing mode in speeded discrimination. Journal of Experimental Psychology, 96, 389-395.

Krueger, L. E. (1973). Effect of irrelevant surrounding material on speed of same-different judgment of two adjacent letters. Journal of Experimental Psychology, 98, 252-259.

LARSEN, A., BUNDESEN, C. (1978). Size scaling in visual pattern recognition. Joumal of Experimental Psychology, 4, 1-20.

Nickerson, R. S. (1972). Binary-classification reaction time: A review of some studies of human information-processing capabilities. Psychonomic Monograph Supplements, 4, 17(Whole No. 65).

Redding, G. M., * Tharp, D. A. (1981). Processing line location and orientation. Joumal of Experimental Psychology: Human Perception \& Performance, 7, 115-129.

SANTEE, J. L., EGETH, H. E. (1980). Selective attention in the speeded classification of multidimensional stimuli. Perception \& Psychophysics, 28, 191-204.

SMITH, A. F. (1986). Strategies and structure in selective attention tasks. Unpublished doctoral dissertation, Yale University, New Haven, CT.

WINER, B. J. (1971). Statistical principles in experimental design. New York: McGraw-Hill.

(Manuscript received December 18, 1987; revision accepted for publication February 22, 1988.) 